\title{
'Diabetes doesn't matter as long as we're keeping traditions alive' a qualitative study exploring the knowledge and awareness of Type 2 diabetes and related risk factors amongst the young Punjabi Sikh population in the UK
}

DOI:

10.1080/13557858.2020.1827141

\section{Document Version}

Accepted author manuscript

Link to publication record in Manchester Research Explorer

Citation for published version (APA):

Sidhu, T., Lemetyinen, H., \& Edge, D. (2020). 'Diabetes doesn't matter as long as we're keeping traditions alive' a qualitative study exploring the knowledge and awareness of Type 2 diabetes and related risk factors amongst the young Punjabi Sikh population in the UK. Ethnicity \& Health. https://doi.org/10.1080/13557858.2020.1827141

\section{Published in:}

Ethnicity \& Health

\section{Citing this paper}

Please note that where the full-text provided on Manchester Research Explorer is the Author Accepted Manuscript or Proof version this may differ from the final Published version. If citing, it is advised that you check and use the publisher's definitive version.

\section{General rights}

Copyright and moral rights for the publications made accessible in the Research Explorer are retained by the authors and/or other copyright owners and it is a condition of accessing publications that users recognise and abide by the legal requirements associated with these rights.

\section{Takedown policy}

If you believe that this document breaches copyright please refer to the University of Manchester's Takedown Procedures [http://man.ac.uk/04Y6Bo] or contact uml.scholarlycommunications@manchester.ac.uk providing relevant details, so we can investigate youeclaim.

\section{OPEEN" ACCESS}


"Diabetes doesn't matter as long as we're keeping traditions alive":

A qualitative study exploring the knowledge and awareness of Type 2

Diabetes and related risk factors amongst the young Punjabi Sikh

population in the UK.

Ethnicity and Health

\author{
Tarnjit Sidhu ${ }^{1}$ \\ Email: tarnjit.sidhu@ @ostgrad.manchester.ac.uk \\ Twitter: @TarnjitSidhu \\ Dr. Henna Lemetyinen ${ }^{1,2}$ \\ Email: henna.lemetyinen@manchester.ac.uk \\ Professor Dawn Edge ${ }^{1,2}$ \\ Email: Dawn.Edge@manchester.ac.uk \\ ${ }^{1}$ School of Health Sciences \\ University of Manchester \\ Oxford Road, Manchester \\ M13 9PL \\ Twitter: @EdgeDawn \\ ORCID: 0000-0003-1139-6613 \\ ${ }^{2}$ Greater Manchester Mental Health NHS Foundation Trust \\ Research \& Innovation \\ Bury New Road \\ Manchester M25 3BL
}

Word count: 6955 


\section{Acknowledgements}

I would like to say a big thank you to all the participants for their invaluable input. Without you all this research would not have been possible. 


\title{
“Diabetes doesn't matter as long as we're keeping traditions alive": A qualitative study exploring the knowledge and awareness of Type 2 Diabetes and related risk factors amongst the young Punjabi Sikh population in the UK.
}

\begin{abstract}
Objectives: It is well known that Sikhs are at an increased risk of Type 2 Diabetes (T2DM) due to a genetic predisposition. High level of education is associated with lower incidence of T2DM. Young Sikhs are well educated compared to other South Asian sub-groups. Despite this, T2DM rates remain high in Sikhs. The uptake of preventative services is also low within Sikhs. At present, no research has been conducted with the young Punjabi Sikh population on diabetes awareness in the UK. To address this gap, this study explores the knowledge and awareness of T2DM and related modifiable risk factors in the UK Punjabi Sikh community. Views surrounding T2DM healthseeking behaviours are also explored.
\end{abstract}

Design: A qualitative design comprising 1-to-1 semi-structured interviews was adopted. Thirteen Punjabi Sikh participants between the ages of 18-30 took part. Participants were recruited through a Sikh temple and University of Manchester Sikh Society using purposive and snowball sampling in West Yorkshire and North West England. Data were analysed using inductive thematic analysis taking a critical realist stance.

Results: Three themes were identified: (1) Perceptions of the causes of T2DM, (2)

Perceptions of factors impacting physical activity and diet, and (3)

Attitudes towards health-seeking behaviours.

Conclusions: The findings show that although well educated, participants overlooked physical activity as contributing factor towards T2DM onset. Additionally, gender and cultural norms 
influenced physical activity and diet, as these are passed through generations to preserve the collectivist Sikh culture. Exploration of health seeking behaviours also found young Punjabi Sikhs perceived the internet to be the preferred tool to seek T2DM information. These findings hold implications for health professionals, as the information and preventative services provided to Sikhs can be tailored to be culturally appropriate and in line with cultural and gender norms, such as bhangra dancing for physical activity.

Keywords: Type 2 diabetes; diabetes; UK; awareness; knowledge; risk factors; South Asian; Sikh; Punjabi Sikh; British Sikh. 


\section{Introduction}

\section{Type 2 Diabetes and the South Asian population}

Type 2 Diabetes (T2DM) is a metabolic disorder characterized by insulin resistance and impaired insulin secretion. The condition has many adverse effects and leads to increased risk for chronic diseases such as cardiovascular disease and atherosclerosis (Ozougwu 2013). In the UK alone, 2.9 million people are diagnosed with diabetes (Health and Social Care Information Centre 2017), of which 90\% have T2DM (Stuckey et al. 2010). The growing rate of T2DM in the UK is a concerning issue for the National Health Service (NHS). T2DM accounts for approximately $10 \%$ of the annual NHS budget at around $£ 8.8$ billion a year, and is projected to account for around 17\% in 2035/2036 (Hex et al. 2012). Hence, T2DM research in the UK is imperative in order to contribute towards preventative measures and reducing healthcare costs.

Furthermore, T2DM onset is associated with modifiable risk factors:

physical inactivity and unhealthy dietary practices. If neglected, they can lead to the development of health complications such as heart disease (Stuckey et al. 2010). With rates of obesity on the rise and the population becoming more sedentary (Health and Social Care Information Centre 2017), the prevalence of T2DM in the UK is increasing (NCD Risk Factor Collaboration 2016).

The increase of T2DM has resulted in a renewed government focus on management and prevention of T2DM in the UK. National Institute for Health and Care Excellence (NICE) have developed public health guidelines for T2DM prevention among high-risk groups and the prevention of 'pre-diabetes' among adults aged 18-74 (NICE 2011). Research shows that ethnicity is a significant influencing factor in health behaviours 
amongst 'at risk' groups (Ibrahim 2013), therefore recommendations include the importance of culturally appropriate interventions for minority groups.

People of South Asian (SA) origin are native to the Indian Subcontinent, which includes India, Pakistan, Bangladesh, Nepal and Sri Lanka (Misra and Shrivastava 2013), and can be identified by their religious sub-groups: Hindu, Sikh and Muslim (Oliffe et al. 2010). SA's are at an increased risk of T2DM both in their home and migrated countries (Unnikrishnan, Gupta, and Mohan 2018). Three major risk factors have been identified as placing SA's at a disproportionately high risk for T2DM: genetic predisposition, poor dietary habits, and low levels of physical activity (Eapen et al. 2009). Due to the genetic predisposition this population hold, it is particularly important that modifiable risk factors are targeted to reduce the risk of T2DM.

\section{A focus on the Sikh population: Sikhs in the UK}

Sikhism is a monotheistic religion underpinned by three core values: making an honest living, engaging in community service (known as seva) and remembering God (Sohi, Singh, and Bopanna 2018). Sikhs belong to a collectivist culture, which are characterized by values of the in-group. These include prioritising the in-group integrity rather than personal goals, focusing on the interdependence of members, and focusing on preserving social relationships within the group (Chadda and Deb 2013). Therefore, the views of members of this in-group (i.e. other Sikhs) are highly valued (Thandi 2018).

According to the 2011 census, there are 432,000 Sikhs in the UK, making up $0.7 \%$ of the population. Fifty-five percent of Sikhs are British born, with 59.4\% of the total UK Sikh population being under the age of 34 (Office for National Statistics 2011). Given that the UK Sikh community is skewed towards young Sikhs, it is important to engage in health 
research with this underrepresented population to encourage positive preventative measures.

Furthermore, research shows that Sikhs that reside in Western countries have a higher risk of T2DM compared to the general population (Patel et al. 1995; Sidhu et al. 2016). Although it is known that individuals of South Asian origin in the UK are 6 times more likely to develop T2DM than their White British counterparts (Stuckey et al. 2010), the latter remains unknown for Sikhs in particular. Approximately $70 \%$ of British born Sikhs between the ages of 18-24 have a graduate qualification/experience of higher education participation therefore are comparatively well-educated compared to other SA sub-groups (Thandi 2018). Despite this, T2DM levels in Sikhs remain high (Sidhu et al. 2016). Thus, it is important to explore gaps in young UK Sikhs' T2DM knowledge and awareness to understand factors contributing towards this prevalence.

British-born Sikhs were included in this study; therefore, their ethnic origin is described as British Sikh. Because British born Punjabi Sikhs have origins in the Northern Indian province of Punjab, the majority of British Sikhs identify as Punjabi (Chanda and Ghosh 2012; British Sikh Report 2017). Subsequently, this research will focus on the Punjabi Sikh population in the UK.

\section{T2DM risk factors: Sikhs and diet}

Dietary behaviours are a modifiable risk factor contributing to the onset of T2DM (Health and Social Care Information Centre 2017). In Sikhism, 'langar' - the meal served to the community by the community is the central aspect of 'seva'. Seva refers to selfless service without any expectation of reward/benefit (Sohi, Singh, and Bopanna 2018). 
The Sikh diet typically includes wheat and vegetable based dishes (Goyan-Kittler and Sucher 1989). Although the use of natural ingredients is common, the accessory foods, such as ghee (clarified butter) and oils that are used to cook the natural ingredients contain a high proportion of fat. As a result, the diet contains high proportions of fats and sugars, and food is typically fried (Varghese and Moore-Orr 2002), making these unhealthy despite the use of vegetables.

Hospitality is fundamental to the consumption of Indian food. Refraining from this carries social consequences such as discrimination from community members (Grace et al. 2008). Grace et al. (2008) found that 'everyday foods' e.g.

plain lentils and rice were not considered appropriate for guests. Instead, 'special foods' e.g. fried snacks were reported as more socially acceptable. Majority of the Indian snacks commonly consumed on momentous occasions are overloaded with ghee or vegetable oils (Gulati and Misra 2017). In a social context, the consumption of these snacks play an important role in the offering and receiving which takes place within the context of 'giftgiving' (Greenhalgh, Helman, and Chowdhury 1998) and is a tradition that has been preserved over time. Hospitality is also linked to social status within the Sikh community (Grace et al. 2008); therefore, it holds importance in Sikh families. Being part of a collectivist (vs. individualistic) culture, where hospitality and food are representative of social status, can make dietary change difficult due to the risk of social rejection.

\section{T2DM risk factors: Sikhs and physical activity}

Physical activity (PA) is defined as any movement of the human body in daily life, such as work, slow walk or participating in sports (WHO 2010). In the UK, 150 minutes of 
moderate activity per week or 75 minutes of vigorous activity per week are recommended to attain the beneficial effects of PA (Department of Health 2010).

In the UK, $17 \%$ of Indians met current PA recommendations (Dowse et al. 1991) compared with 16\% of Pakistanis, 10\% Bangladeshis (Sarkar et al. 2017) and 60\% of the adult UK population (Scholes 2017). The rate of PA in UK Sikhs specifically is unknown. Few studies have explored PA levels in Sikhs exclusively, however some have included Sikhs as SA sub-group (Williams et al. 2011).

Due to the views of others in the in-group being highly regarded, this may make it difficult for Sikhs to engage in activities outside social norms within the collectivist culture, such as PA.

\section{T2DM and Punjabi Sikhs in the UK}

Due to the vastly growing cultural diversity in the UK, it is important to research UK Sikhs, as they are currently under-researched.

Following migration from India, the North of England became a place of residence for many Punjabi Sikhs (British Sikh Report 2017). Although diabetes research has been undertaken in central England ('Midlands') and in London (Mather and Keen 1985; Sidhu et al. 2016) to present knowledge there is no evidence for research being undertaken in the North of England. Therefore, the present research will recruit participants from West Yorkshire and North West England. Furthermore, since migration, Sikhs have experienced relative social mobility with $69 \%$ currently economically active (second to Hindus with 70\%) (Thandi 2018). Despite this, rates of T2DM remain high in Sikhs in the UK (Sidhu et al. 2016). A factor contributing towards this may be the understanding of T2DM and related risk factors. 


\section{Aims}

This research aimed to explore the following within young Punjabi Sikhs in the UK:

(1) Their knowledge and awareness of T2DM and related risk factors (diet and PA).

(2) Their views towards health-seeking behaviours for T2DM related information.

From this, gaps in T2DM knowledge can be identified, as well as factors impacting related risk factors. This can lead to better understanding of the specific health needs of this population to put preventative measures in place and allow appropriate health information to be communicated with Sikhs.

Health-seeking behaviours are defined as any activity undertaken to find an appropriate remedy for a health problem (Olenja 2003). Research shows Sikhs are more likely to have limited availability to healthcare resources, alongside having a lower than average uptake of preventative healthcare services (Sidhu et al. 2016). Based on this, a secondary aim will be explored through a hypothetical question asked at the end of the interview around participants' health-seeking behaviours. The potential implications of this may facilitate tailoring support services for young Sikhs.

\section{Materials and methods}

\subsection{Design}


This study adopted a qualitative design. This study adopted a qualitative, phenomenological design. The goal of phenomenology is to describe phenomena from the perspectives of those who have experienced it (Neubauer, Witkop, and Varpio 2019). This approach was therefore appropriate for the present study, which sought to explore the views and experiences of Young Sikhs in relation to diabetes for the first time. This exploratory study used semi-structured interviews to explore the knowledge and awareness Punjabi Sikh participants held regarding T2DM and its related modifiable risk factors, specifically, PA and diet. Interviews were conducted due to their flexibility in exploring the views and experiences of participants (Braun and Clarke 2006). The flexibility of this approach encourages elaborative responses and ensures the topic in question is being addressed whilst maintaining a suitable structure. As no literature on young UK Sikhs views surrounding T2DM has been published to the authors' knowledge, qualitative approach was deemed most appropriate as the starting point.

\subsection{Recruitment \& Participants}

Thirteen participants from the Punjabi Sikh community were recruited through purposive sampling techniques (Devers and Frankel 2000). This technique aims to generate in-depth understanding of the research topic due to participants providing 'information rich' data (Patton 2002).

Participants were recruited through a Sikh temple in West Yorkshire $(n=7)$ and Sikh Society at The University of Manchester (UoM) $(n=6)$. A poster advert was shared in the temple alongside an informal workshop aiming to address any questions. A poster was also taken to a UoM Sikh Society event where the first author presented the research and 
answered questions. People who were interested in participating emailed the first author for an information sheet.

Additionally, snowball sampling was used as participants that were interested in taking part recommended other Punjabi Sikhs to partake in the study. Although purposive sampling helps recruit participants, snowball sampling has been seen as an effective technique to engage the 'hard-to-reach' population in a culturally acceptable manner (Sadler et al. 2010). Data collection took place between December 2018 and May 2019, by when data saturation had been reached (Braun and Clarke 2006).

The inclusion criteria comprised age (18-30 years), and self-identification as Punjabi Sikh. Due to informed consent being essential before participation, the exclusion criteria limited individuals who were unable to read and write. Other religious or ethnic groups were also excluded due to the research requirements.

\subsubsection{Ethical Approval}

Ethical approval was granted by The University of Manchester Research Ethics Committee prior to recruitment (Ref: 2018-4148-7520).

\subsection{Materials}

An interview guide was developed based on previous literature and theory. Concepts of The Health Belief Model (HBM) (Rosenstock 2000) informed the interview guide (available upon request). The questions explored respondents' knowledge and awareness surrounding T2DM and related modifiable risk factors: PA and diet. Research shows Sikhs have a lower than average uptake of preventative healthcare services (Sidhu et al. 2016), therefore the interview guide also included hypothetical questions surrounding T2DM 
related health-seeking behaviours. A pilot interview (excluded from the dataset) was conducted to allow appropriate modifications to be made to the content and language of the guide prior to data collection.

The Health Belief Model (HBM)

The Health Belief Model (HMB) is a well-validated model commonly used to explain health behaviours and the lack of preventative actions taken (e.g. Guvenc, Akyuz, and Açikel 2011), which is often observed in Sikhs (Sidhu et al. 2016). It has been previously applied to explore cervical and breast cancer screening (Darvishpour, Vajari, and Noroozi 2018; Sadat 2012).

This model proposes that the likeliness of an individual performing specific health behaviours is measured through six constructs, illustrated in Figure 1. In the context of T2DM, the model suggests that individuals are most likely to adopt health behaviours when they believe that (1) they are susceptible to T2DM, (2) it will have severe consequences for their health, (3) the benefits of unhealthy behaviours outweigh the costs, and (4) they have the necessary skills to carry out the health behaviours (healthy diet and PA). Adopting the HBM as a framework will allow exploration the perceptions of T2DM and related risk factors. Although this model has not been culturally adapted, the constructs have been found to be applicable in a range of populations such as Iranian women and breast cancer (Taymoori and Berry 2009).

[Figure 1 to be inserted here] 


\subsection{Procedure}

Participants, who expressed interest in taking part, were provided with a participant information sheet via e-mail or in person in West Yorkshire or North West England. Participants were given an opportunity to ask questions prior to obtaining a written informed consent.

Thirteen participants were interviewed at a mutually convenient location (e.g. a meeting room at the Sikh Temple or the University of Manchester). The first author conducted the audio-recorded, semi-structured interviews, lasting up to one hour. Data were stored on a password-protected computer. Audio recordings were transcribed verbatim by the first author and a pseudonym was allocated to each participant to ensure anonymity. Verbatim transcription is beneficial as the researcher had first-hand knowledge from involvement in the interview process, bringing researchers closer to their data. This is advantageous for data analysis (Halcomb and Davidson 2006).

\subsection{Data Analysis}

The data were analysed using thematic analysis (TA). TA is a qualitative approach to identifying patterns ('themes'). These themes, which emerge without a priori assumptions about the data, are analysed and interpreted within the context of the research (Braun and Clarke 2006). TA was appropriate due to its flexible nature (Braun and Clarke 2006). An essentialist approach was adopted in order to tell the 'story of the data' (Braun and Clarke 2013). An inductive form of TA, which assumes no prior themes or framework of the data, was applied to allow emergence of 'data-driven' themes (Braun and Clarke 2006). Codes 
were identified on a semantic level, therefore coding "mirrored participants' language and concepts" (Braun and Clarke 2013, 207).

The ontological stance taken was critical realism, which is consistent with an inductive TA approach (Braun and Clarke 2006). Critical realism holds that knowledge or 'truth' can exist and be shared by individuals, however each individual's experience of truth will be influenced by their own construction of that truth (Robson 2002). This stance was deemed appropriate as participants' knowledge and awareness of T2DM and related risk factors could only be accessed through their unique perceptions. The epistemological stance taken was 'contextualism'. This perspective does not assume a single reality; instead it recognises that knowledge emerges from contexts (Madill, Jordan, and Shirley 2000).

Six stages of thematic analysis were conducted as described by Braun and Clarke (2006). These are shown in Table 1.

[Table 1 to be inserted here]

\subsection{Trustworthiness and Rigour}

To ensure trustworthiness, the second author (HL), experienced with qualitative methods, familiarised herself with a transcript. This is seen as a valuable strategy to ensure rigour in thematic analysis (Barbour 2002). Whilst being blinded to the coding, independent coding of the transcript was undertaken. The independently generated codes for the transcript were crosschecked, which resulted in the identification of minute differences between codes. This was due to the wording used to describe codes, therefore a discussion amongst HL and TS confirmed agreement of the content of codes. Peer-examination of data increases the credibility and dependability of the research (Lincoln and Guba 1985). 
Furthermore, member checking was conducted to ensure the results were credible. This technique consists of testing the data, analytic categories, interpretations and conclusions with informants, which ensures the researcher has accurately translated the informants' viewpoints into data (Lincoln and Guba 1985). A key informant was identified in the interviewing process due to his in-depth knowledge on Sikhism and involvement in the Sikh community. Upon consent, the key informant was contacted via email following phase 3 (searching for themes) and phase 5 (defining and naming themes) of TA, and was asked for input regarding the codes generated. Following discussion, the key informant agreed the proposed codes reflected views accurately (Lincoln and Guba 1985).

\subsection{Reflexivity}

Reflexivity refers to the process of acknowledging the researchers' subjective experiences and how this may influence the process of analysis and data interpretation (Mosselson 2010). Insider status refers to the researcher sharing a common ethnic and cultural background as the participants (Merton 1972). The researcher (TS) held insider status, as she and the participants identified as Punjabi Sikh. Being part of the Sikh community, and being an individual who attends the Sikh temple regularly, the researcher had insight on the dishes served at the temple and in Sikh families, alongside awareness of the high prevalence of T2DM in Sikhs. Having social connections within the Sikh community meant that during recruitment, purposive sampling was able to take place through the Sikh temple and UoM Sikh Society.

Holding insider status meant the researcher had in-depth understanding of the traditions and norms of Sikhs, and had cultural intuition of the topics being discussed. This benefits rapport building, as the researcher was able to identify with the participant and probe 
appropriately. As such, the researcher acknowledges this shared background may have had an impact on interpretation of the data. This was balanced by having a transcript crosscoded by HL, a researcher who held outsider status (Merton 1972). Care was taken to minimize the extent to which the researcher's insider status impacted analysis and the process of coding and theme-formation was data-driven.

\section{Results}

Thirteen British-born Punjabi Sikh participants between the ages of 18-30 years were recruited. Seven participants were recruited through a Sikh temple, and six were recruited through the UoM Sikh Society. Twelve out of thirteen participants were living at their family home. Participants were predominantly female $(n=11 / 13 ; 85 \%)$ (See Table 2.). Consistent with the common demographic of young Sikhs, all participants were educated to at least degree level.

[Table 2 to be inserted here]

\section{Themes}

Three key themes were identified: (1) Perceptions of the causes of T2DM, (2) Perceptions of the factors impacting diet and PA, and (3) Attitudes towards health-seeking behaviours.

Theme 1: Perceptions of the causes of T2DM 
When discussing the factors causing T2DM, the majority of participants $(n=9)$ expressed an understanding of their risk of developing T2DM being higher than average due to a genetic pre-disposition. Participants perceived excess of sugar intake as a primary behavioural cause for Sikhs: 'So I think we [Sikhs] are more likely to get it 'cause of our genes but then it's when people eat too much sugary foods' (Priya)

Most participants $(\mathrm{n}=10)$ also revealed that in their family, 'the older generation call it [T2DM] sugar' (Manvir). This use of language by the older generation is likely to have impacted the perceptions of the participants, as an emphasis has been placed on sugar intake in diet as a cause of T2DM. Further supporting this, when asked what advice should be given to a person with T2DM, Monica replied with 'cutting out anything that contains sugar'.

Comparatively, Hardeep recognised that as well as foods labelled as high in sugar, intake of 'hidden' sugars in food are also relevant: 'There are hidden sugars that you maybe don't realise you're eating so it could be you're getting sugar from other foods you wouldn't have thought you're getting sugar from' (Hardeep).

In comparison to diet, lack of PA was not perceived as a significant risk factor for the onset of T2DM:

I'd say it's mostly diet to be honest 'cause it's just eating too much sugar isn't it [...] I don't think exercise is as responsible cause' when I think of diabetes I just think of sugar and food it's what goes into our bodies (Jas) 
Sukhraj agreed with this, as he explained that growing up in a Punjabi Sikh family sugar intake was emphasised therefore he perceived diet as more important than exercise for T2DM onset:

Yeah it's cause growing up I knew sugar was related to diabetes cause' mum would say I'm not eating Indian sweets cause' I've got sugar I kind of know exercise is related cause' you should be healthy and all that but even then I think it's more to do with what you eat (Sukhraj).

\section{Theme 2: Perceptions of the factors impacting diet and PA}

Dietary practices and levels of PA were impacted by gender and cultural norms within participants.

Sub-theme 2.1. Cultural norms: the importance of hospitality and family reputation

The perception that hospitality is central to social gatherings was commonly held among participants: 'Hospitality is a big thing for Sikh families' (Anita), with participants explaining that hospitality was perceived to be linked to showing respect and welcoming guests into the home:

I guess it's just in our culture it's just what we do it's how we invite people make them feel welcomed in our house having a lot of food it's just some form of respect that when people come to your house it shows that you're happy to have them there by having all this food (Raji). 
Participants expressed that in Punjabi Sikh families, food and food preparation were closely linked to family reputation, which was perceived as important:

I think it's all just about food and drinks and whether the food's nice and made properly so like fried and the more food and drinks you have the more reputable you are so reputation of the people is higher and the respect is higher [...] I think it shows they're more affluent even (Karam).

Alongside hospitality, the type of foods served as events also poses risk to the reputation of a Punjabi Sikh family, as 'rich' Indian foods are expected to be served. This was perceived to hold importance over being healthy:

Indian food is very rich and it's more about flavour than the health benefits of it so you have to serve the rich foods cause' it plays a big role and it's about reputation as well so the better the food tastes the more respected you are [...] it's like it's always a competition between the aunties on who makes the best curry (Nisha).

Although the younger generation are attempting to make changes to dietary practices, this is viewed as challenging due to the perception of food preparation being related to respect for elders. Cultural traditions that had been passed through generations held significant importance over T2DM:

Sometimes I want to change some ingredients but I think the older generation would see it as being disrespectful because they're really focused on feeding the family and impressing people so they wouldn't be happy if you try change things and try and adapt recipes just because you're trying to be a bit more healthy [...] it's how we're taught we should do 
things and diabetes doesn't matter as long as we're keeping traditions alive even if it means we're unhealthy cause' of it (Maya).

Sub-theme 2.2. Gender norms: Expectations of women in the family

Majority of the participants were female and commonly expressed experiences related to PA and dietary practices in a Punjabi Sikh family. Female participants spoke about being taught how to cook from a young age from older family members. Hence, the females of every generation in a family were predominantly in control of the cooking:

Being in a Sikh family you are not only taught from your mother but by your grandma and your aunties 'cause we're the ones who cook for our families so we're show growing up how much oil and spices to add to food to make it taste good (Monica).

Additionally, participants discussed prioritizing family over PA as it was 'not the Sikh thing to do' (Priya). Participants expressed that the elders in their family perceived exercise to be a 'waste of time' (Manvir) and saw cooking as a priority over PA as preparation for marriage. This discouraged them from engaging in PA: 'I try and stay healthy and go to the gym but sometimes my mum doesn't like it when I go 'cause she'll say when you're married you'll need to cook gym isn't going to help you' (Sonia).

Conversely, participants described bhangra, a traditional dance originating from India, as being a form of PA they had enjoyed and that had been better accepted by the elders in the family than other forms of exercise: 
Bhangra I can do it at weddings but I'd love to learn properly 'cause it's part of our culture and our roots you know our identity [...] I think my mum would love it too then she can show off her moves at weddings (Karam).

One participant who had previously danced in Bhangra teams at university discussed how female only bhangra teams were on the rise in the central England region ('midlands') which focused on providing females a safe space to exercise and have fun:

I did bhangra at uni and my mum and dad loved it 'cause I was doing something that was related to being Punjabi [...] there's loads of girls teams now more in the Midlands but we need them here too 'cause we just used to have fun and exercise and our parents were happy too (Amrit).

Hardeep agreed that females should be encouraged to engage in a form of exercise perceived as appropriate such as bhangra dancing, which was traditionally seen as a predominantly male activity. He explains that this should be encouraged due to its cultural relevance:

I know boys used to do bhangra back in the day but bhangra is from our culture so they [females] should be more encouraged to do that and if they're not encouraged to do that they're gonna be less likely to take up anything else outside of our culture

(Hardeep).

\section{Theme 3: Attitudes towards health-seeking behaviours}

Attitudes towards health-seeking behaviours was the final prominent theme across the interviews. The two main resources that were referred to when exploring T2DM health- 
seeking behaviours were visiting a health professional, i.e. a general practitioner, and using the Internet.

Sub-theme 3.1. Using the Internet vs. visiting a healthcare professional

The majority of interviewees expressed that if they required T2DM information, they would prefer to use the internet over visiting a health professional due to the perception of the internet being a convenient and easy tool compared to the effort taken to visit a GP:

I'd just use the internet cause' you can find out anything on the internet now and there's specific websites for diabetes I just find going to the doctors is effort and it takes ages to make an appointment but you can use the internet whenever you want so it's easy (Anita).

Participants expressed a number of reasons as to why they perceived using the Internet for T2DM information as preferable compared to visiting a GP. Firstly, participants perceived GP's as lacking cultural awareness specific to Sikhs:

They [GP's] don't get our cultural traditions like what we eat and why we eat it like we have like Indian sweets as a gift and they probably say don't have that but we have to have it 'cause it's part of a tradition and we can't replace that (Sonia).

Secondly, interviewees expressed that health professionals used language perceived as 'complicated medical terminology' (Raji), leaving participants feeling worse following a visit: 'They say all these technical terms and sometimes you think you're going there to help yourself but it makes you feel worse at the end of it 'cause you don't understand' (Maya) 
Hence, some participants expressed a preference towards using the Internet for T2DM information, as this was independent and less complicated than information provided for a GP:

I'd use the Internet because you can do your own research and learn a lot more about the disease in depth and get a better understanding in a way that you understand 'cause the GP can complicate things with the words they use (Jas).

Additionally, some interviewees discussed their experiences of visiting Sikh doctors who they assumed would give tailored and helpful information however experienced the opposite, as the doctor avoided conversation around difficult issues:

'I thought we [Sikhs] should see Sikh doctors 'cause they get it but when I've seen Sikh doctors it's like they don't wanna talk about difficult stuff' (Karam).

Manvir agreed and expressed that she perceived Sikh doctors as dismissive compared to non-Sikh doctors based on a previous experience, and had viewed this to be the result of their shared background: 'I'd want to see a white doctor 'cause Ifeel like when I've seen a Sikh doctor they don't take things seriously when they see another Sikh person' (Manvir).

\section{Discussion}

It was found that participants were generally knowledgeable of the genetic and diet related risk factors for T2DM, however were less knowledgeable of PA being a T2DM risk factor. Furthermore, participants perceived gender and cultural norms to impact their diet and level of PA. More specifically, hospitality and providing 'rich foods' was linked with family reputation (cultural norm), and females were expected to prioritize learning how to 
cook for the family over engaging in PA (gender norm). Both of these factors disregarded PA, therefore are likely to have impacted participants' perceptions regarding T2DM causation, as PA was the only risk factor that was less understood. Moreover, exploration of attitudes towards health-seeking behaviours suggested a preference towards Internet use for T2DM information in compared to visiting a health professional such as a GP.

Cultural factors e.g. the importance of hospitality and family reputation were perceived to impact T2DM risk factors. This reflects previous research by Sidhu et al. (2016) who found that Sikhs were expected to conform to established cultural values alongside being hospitable towards guests as this was related to Seva: the provision of selfless service. Offerings of food were seen to be a symbolic gesture of family status (Sidhu et al. 2016). This finding is also consistent with research conducted by Grace et al. (2018) who found that in Sikh families, hospitality and the provision of 'rich foods' such as fried snacks was linked to high social status.

Research shows the younger Sikh generation is highly influenced by older members of the family, with cultural norms being passed down through generations (Singh 2010). This is likely to be due to cultural transmission, especially of food habits, as it brings cohesion to the identity of groups (Quintero-Angel, Mendoza, and Quintero-Angel 2019). Food constitutes an important element among Punjabi Sikhs as sharing allows them to strengthen the ties that generate social cohesion. This may explain the emphasis of hospitality and 'rich' foods over health preservation in Punjabi Sikhs.

Additionally, gender norms were seen to impact T2DM risk factors, as gender expectations prevented women from partaking in PA. Although Sikhism promotes equality of genders (Singh 2010), it is possible that Sikhs who have migrated to Westernized societies aim to keep gender norms consistent to stay in line with their cultural identity 
(Narayan 1995). This may explain why parents discouraged females from engaging in PA. Although specific research with Sikhs in the UK has not been conducted, research with SA women supports this: Lawton et al. (2008) found that the culturally desirable focus of a woman was perceived to be solely on the family, and activities such as exercising can be viewed as selfish. Collectivist cultures highly regard the views on in-group members, and from a young age Sikhs are socialised into Indian collectivist cultural values of duty, cooperation and obedience (Triandis, Chen, and Chan 1998). Women may therefore be discouraged from taking part in PA due to concerns regarding stigma from fellow community members.

Furthermore, a form of PA that is culturally relevant to Punjabi Sikhs is bhangra dancing. Bhangra dancing is a folk dance originated in Punjab. It is typically representative of Sikh Punjabi cultural identity (Leante 2012). The present research found bhangra dancing was encouraged by the older Sikh generation as a form of exercise, despite the gender expectations of women (Lawton et al. 2008). This bridging of the intergenerational gap was likely to be due to the cultural significance of bhangra to Punjabi Sikhs (Leante 2012). Thus, our findings imply that forms of PA that are also an expression of culture and/or tradition might be more acceptable to older Sikh generations compared to PA that might be viewed purely as physical exercise (e.g. going to the gym).

The present study also found that participants perceived the Internet as a suitable tool for seeking T2DM information compared to visiting a GP due to the complicated terminology used by professionals, and the lack of cultural awareness specific to Sikhs. It is commonly assumed that ethnic matching of young clients and health professionals is beneficial for positive health outcomes due to a shared cultural understanding (Jerrel 1998). The present study however challenges this, as several participants expressed a preference 
towards visiting health professionals of a different ethnicity to their own due to experiences of Sikh health professionals being dismissive.

\subsection{Strengths and limitations}

Our study is the first in the UK focusing on the young Sikh Punjabi community.

Previous research has included an older Sikh sample (Sidhu et al. 2016), or South Asians as heterogeneous group (Lawton et al. 2008), however the present study addresses a gap in the literature by including the young Sikh Punjabi generation and exploring their T2DM related knowledge and awareness. Furthermore, due to the majority of the UK Sikh population identifying as Punjabi Sikh (Chanda and Ghosh 2012), the sample is representative of the young Sikh population in the UK (Chanda and Ghosh 2012).

Purposive and snowball sampling resulted in thirteen participants, which is sufficient for thematic analysis (Braun and Clarke 2006). Snowball sampling allowed access to this 'hard-to-reach' population. Although purposive and snowball sampling increase accessibility in so called 'hard-to-reach' populations, it is important to note that these sampling techniques also introduce a degree of selection bias (Tongco 2007). For example, participants agreeing to take part may be interested in health and diabetes, and may recommend people they know are also interested in these topics, decreasing the generalisability of the findings.

Furthermore, one participant acted as a key informant (Sadler et al. 2010). Allocating a key informant (Hardeep) allowed a key participant to be involved with the study and ensure that the 'final presentation of the data reflected the experience accurately' (Lincoln and Guba 1985). The role of a key informant is to be representative of the views of the group and be able to provide insight into the views of the community (McKenna and 
Main 2013). Hardeep met the criteria of key informants suggested by Tremblay (1957), as he was a significant member of the Sikh community through volunteering at the Sikh temple, had knowledge relevant to the study and was willing to share this, and communicated this effectively. Furthermore, the present study had eleven female and two male participants. Although the male participants provided valuable insight, the results could be skewed towards being representative of female Punjabi Sikhs, limiting generalisation.

\subsection{Implications for policy/practice}

(1) Our findings suggest that participants lacked knowledge regarding the contribution of PA to T2DM development. Although research exploring young people's knowledge about T2DM is limited in the UK, these findings have been replicated in the United Arab Emirates. It was found just over half of the students in this study could link obesity and physical inactivity as risk factors for developing T2DM (Khan et al. 2012). Furthermore, young Punjabi Sikhs are perceived as well educated (Thandi 2018), therefore would be expected to have better T2DM knowledge than reported. These results hold implications for health professionals, as an emphasis should be placed on PA as a modifiable risk factor for T2DM when communicating T2DM information.

(2) Healthcare professionals can work towards incorporating culturally appropriate alternatives for PA such as bhangra dancing into preventative services, or make recommendations on home-based PA to ensure it does not clash with gender expectations. This can help inform culturally appropriate interventions by professionals to pre-diabetic and diabetic patients. 
(3) Young Sikhs expressed that language used by GP's was 'complicated'. Given the education level of participants, these findings are surprising. These hold implications for practice, as health professionals should be mindful and use lay terms when communicating with young Sikhs to assure understanding of health information.

(4) Food practices are passed through generations to preserve the identity of Punjabi Sikhs (Singh 2012). This has implications for healthcare professionals as the concept of portion control could be introduced to preventative services rather than replacement of foods due to food being linked to cultural identity.

\subsection{Future research}

As the present study consisted of a predominantly female sample, future research should be conducted with young male Sikhs to explore whether views are consistent across genders due to Sikh males being underrepresented in this study. Furthermore, findings suggest that although ethnic matching is suggested by Jerrel (1998), young Sikhs may prefer not to be ethnically matched to a Sikh health professional. Further research is required with Sikhs to explore this finding in detail alongside research with Sikh healthcare professionals to explore this dynamic. This can work towards ensuring positive changes are made to further support the young Sikh community.

\subsection{Conclusions}

The present study aimed to explore the knowledge and awareness of T2DM and related modifiable risk factors within young Punjabi Sikhs. The study also explored their attitudes 
towards health-seeking behaviours. Although all participants were educated to degree level, awareness of PA being a risk factor for T2DM was lacking.

Furthermore, it was apparent that cultural and gender norms were passed down through generations. PA was discouraged for females (gender norm), and hospitality and diet were important in representing family reputation (cultural norm). Little emphasis was placed on PA in comparison to diet. Furthermore, Young Sikhs also showed a preference towards using the Internet for T2DM information seeking as opposed to visiting a GP, as language used by the GP was perceived as complicated. These results suggest that although young Sikhs are seen as well educated, knowledge regarding T2DM risk factors is lacking. External factors such as cultural and gender norms preserve the collectivist nature of Sikhs. Further understanding of cultural and gender expectations of the young Sikh community is required by health professionals to enable culturally specific recommendations to be made, for example incorporating bhangra dancing, a culturally appropriate form of PA into PA recommendations. Finally, GP's are advised to use lay terms when communicating health information with Sikhs to increase use of preventative healthcare services in young Sikhs.

Funding details. This research was conducted as part of a research degree, and received no funding.

Disclosure statement. The authors declare no conflicts of interest.

Data availability statement. Data are available from the corresponding author upon request.

Data deposition. N/A

Supplemental online material. N/A 


\section{References}

Barbour, R. S. 2002. "Checklists for Improving Rigour in Qualitative Research: A Case of the Tail Wagging the Dog?" BMJ. doi:10.1136/bmj.322.7294.1115.

Braun, Virginia, and Victoria Clarke. 2006. "Using Thematic Analysis in Psychology." Qualitative Research in Psychology. doi:10.1191/1478088706qp063oa.

Braun, Virginia, and Victoria Clarke. 2013. "Quality Criteria and Techniques for Qualitative Research.” In Successful Qualitative Research: A Practical Guide for Beginners.

British Sikh Report. 2018. An insight into the British Sikh Community. http://www.britishsikhreport.org/wp-content/uploads/2017/03/British-SikhReport-2017-Online.pdf

Chadda, Rakesh K, and Koushik Sinha Deb. 2013. "Indian Family Systems, Collectivistic Society and Psychotherapy.” Indian Journal of Psychiatry. doi:10.4103/00195545.105555. 
Chanda, Rupa, and Sriparna Ghosh. 2012. "The Punjabi Diaspora in the UK: An Overview of Characteristics and Contributions to India." SSRN Electronic Journal. doi:10.2139/ssrn.2179734.

Danaei, Goodarz, Mariel M. Finucane, Yuan Lu, Gitanjali M. Singh, Melanie J. Cowan, Christopher J. Paciorek, John K. Lin, et al. 2011. "National, Regional, and Global Trends in Fasting Plasma Glucose and Diabetes Prevalence since 1980: Systematic Analysis of Health Examination Surveys and Epidemiological Studies with 370 Country-Years and $2 \cdot 7$ Million Participants." The Lancet. doi:10.1016/S0140-6736(11)60679-X.

Department of Health. 2010. "The NHS Outcomes Framework 2011 / 12." Framework.

Devers, K. J., and R. M. Frankel. 2000. "Study Design in Qualitative Research - 2: Sampling and Data Collection Strategies." Education for Health.

Dowse, G. K., P. Z. Zimmet, H. Gareeboo, K. G.M.M. Alberti, J. Tuomilehto, C. F. Finch, P. Chitson, and H. Tulsidas. 1991. “Abdominal Obesity and Physical Inactivity as Risk Factors for NIDDM and Impaired Glucose Tolerance in Indian, Creole, and Chinese Mauritians.” Diabetes Care. doi:10.2337/diacare.14.4.271.

Eapen, D, G L Kalra, N Merchant, A Arora, and B V Khan. 2009. "Metabolic Syndrome and Cardiovascular Disease in South Asians." Vascular Health and Risk Management. 
Goyan-Kittler, P, and K Sucher. 1989. "Food and Culture in America: A Nutrition Handbook.” 1989, Xiii + 384 Pp.

Grace, Clare, Reha Begum, Syed Subhani, Peter Kopelman, and Trisha Greenhalgh. 2008. "Prevention of Type 2 Diabetes in British Bangladeshis: Qualitative Study of Community, Religious, and Professional Perspectives.” BMJ (Clinical Research Ed.). doi:10.1136/bmj.a1931.

Greenhalgh, T., C. Helman, and A M.'m. Chowdhury. 1998. "Health Beliefs and Folk Models of Diabetes in British Bangladeshis: A Qualitative Study.” BMJ. doi:10.1136/bmj.316.7136.978.

Gulati, S., and A. Misra. 2017. "Abdominal Obesity and Type 2 Diabetes in Asian Indians: Dietary Strategies Including Edible Oils, Cooking Practices and Sugar Intake.” European Journal of Clinical Nutrition. doi:10.1038/ejcn.2017.92.

Guvenc, Gulten, Aygul Akyuz, and Cengiz Han Açikel. 2011. "Health Belief Model Scale for Cervical Cancer and Pap Smear Test: Psychometric Testing.” Journal of Advanced Nursing. doi:10.1111/j.1365-2648.2010.05450.x.

Halcomb, Elizabeth J., and Patricia M. Davidson. 2006. "Is Verbatim Transcription of Interview Data Always Necessary?" Applied Nursing Research. doi:10.1016/j.apnr.2005.06.001. 
Health and Social Care Information Centre. 2017. "Quality and Outcomes Framework Prevalence, Achievements and Exceptions Report: England, 2016-17." NHS Digital. doi:ISBN 978 - 1 - 78734 - 160 - 9.

Hex, N., C. Bartlett, D. Wright, M. Taylor, and D. Varley. 2012. "Estimating the Current and Future Costs of Type1 and Type2 Diabetes in the UK, Including Direct Health Costs and Indirect Societal and Productivity Costs." Diabetic Medicine. doi:10.1111/j.1464-5491.2012.03698.x.

Ibrahim, Said A. 2013. "Critical Perspectives on Racial and Ethnic Differences in Health in Late Life.” Annals of Internal Medicine. doi:10.7326/0003-4819-1421-200501040-00023.

Leante, Laura. 2012. "Shaping Diasporic Sounds: Identity as Meaning in Bhangra." The World of Music - Readings in Ethnomusicology.

Lincoln, Yvonna S, and Egon G Guba. 1985. "Establishing Trustworthiness. Naturalistic Inquiry.” In Naturalistic Inquiry.

Madill, Anna, Abbie Jordan, and Caroline Shirley. 2000. “Objectivity and Reliability in Qualitative Analysis: Realist, Contextualist and Radical Constructionist Epistemologies.” British Journal of Psychology. doi:10.1348/000712600161646. 
Mather, Hugh M., and Harry Keen. 1985. "The Southall Diabetes Survey: Prevalence of Known Diabetes in Asians and Europeans.” British Medical Journal (Clinical Research Ed.). doi:10.1136/bmj.291.6502.1081.

Merton, Robert K. 1972. "Insiders and Outsiders: A Chapter in the Sociology of Knowledge.” American Journal of Sociology. doi:10.1086/225294.

Misra, Anoop, and Usha Shrivastava. 2013. "Obesity and Dyslipidemia in South Asians.” Nutrients. doi:10.3390/nu5072708.

Mosselson, Jacqueline. 2010. "Subjectivity and Reflexivity: Locating the Self in Research on Dislocation.” International Journal of Qualitative Studies in Education. doi:10.1080/09518398.2010.492771.

Nam, and Han Cho. 2018. "Q\&amp;A: Key Points for IDF Diabetes Atlas 2017 Q.” Diabetes Research and Clinical Practice. doi:10.1016/j.diabres.2018.01.005.

Narayan, Uma. 1995. "Eating Cultures: Incorporation, Identity and Indian Food." Social Identities. doi:10.1080/13504630.1995.9959426.

NCD Risk Factor Collaboration. 2016. "Worldwide Trends in Diabetes since 1980: A Pooled Analysis of 751 Population-Based Studies with 4.4 Million Participants NCD Risk Factor Collaboration (NCD-RisC) Europe PMC Funders Group.” Lancet. doi:10.1016/S0140-6736(16)00618-8. 
National Institute for Health and Care Excellence. 2011. "Type 2 Diabetes Prevention: Population and Community-Level Interventions." NICE Guidelines [PH35].

Office for National Statistics, and Office for National Statistics. 2011. "Census 2011." Census.

Olenja, J. 2003. "Editorial: Health Seeking Behaviour in Context." East African Medical Journal.

Oliffe, John L., Suki Grewal, Joan L. Bottorff, Jasvinder Dhesi, H. Bindy, K. Kang, Amanda Ward, and T. Gregory Hislop. 2010. "Masculinities, Diet and Senior Punjabi Sikh Immigrant Men: Food for Western Thought?” Sociology of Health and Illness. doi:10.1111/j.1467-9566.2010.01252.x.

Ozougwu, Ozougwu. 2013. "The Pathogenesis and Pathophysiology of Type 1 and Type 2 Diabetes Mellitus.” Journal of Physiology and Pathophysiology. doi:10.5897/jpap2013.0001.

Patton, Michael Quinn. 2002. "Two Decades of Developments in Qualitative Inquiry: A Personal, Experiential Perspective." Qualitative Social Work. doi:10.1177/1473325002001003636.

Quintero-Angel, Mauricio, Diana Marcela Mendoza, and David Quintero-Angel. 
2019. "The Cultural Transmission of Food Habits, Identity, and Social Cohesion:

A Case Study in the Rural Zone of Cali-Colombia." Appetite.

doi:10.1016/j.appet.2019.04.011.

Robson, Colin. 2002. Real World Research. 2nd. Edition. Blackwell Publishing. Malden.

Rosenstock, Irwin M. 2000. “Health Belief Model.” In Encyclopedia of Psychology, Vol. 4. doi:10.1037/10519-035.

Sadler, Georgia Robins, Hau Chen Lee, Rod Seung Hwan Lim, and Judith Fullerton. 2010. "Recruitment of Hard-to-Reach Population Subgroups via Adaptations of the Snowball Sampling Strategy.” Nursing and Health Sciences. doi:10.1111/j.14422018.2010.00541.x.

Sarkar, S., B. Ellahi, F. B. Zotor, and P. Amuna. 2017. "Decreasing Physical Activity Levels across Religious Sikh Male South Asian Migrant Population in Kent, UK: A Public Health Concern." Journal of Health Management. doi:10.1177/0972063417727612.

Darvishpour, Azar, Soheila Mazloum Vajari, and Sara Noroozi. 2018. "Can Health Belief Model Predict Breast Cancer Screening Behaviors?” Open Access Macedonian Journal of Medical Sciences. doi:10.3889/oamjms.2018.183.

Khan, Nelofer, Kadayam G. Gomathi, Syed Ilyas Shehnaz, and Jayakumary 
Muttappallymyalil. 2012. "Diabetes Mellitus-Related Knowledge among University Students in Ajman, United Arab Emirates." Sultan Qaboos University Medical Journal. doi:10.12816/0003144.

Lincoln, Yvonna S, and Egon G Guba. 1985. "Establishing Trustworthiness. Naturalistic Inquiry.” In Naturalistic Inquiry.

Neubauer, Brian E., Catherine T. Witkop, and Lara Varpio. 2019. "How Phenomenology Can Help Us Learn from the Experiences of Others." Perspectives on Medical Education. doi:10.1007/s40037-019-0509-2.

Patel, D. J., M. Winterbotham, R. P. Britt, G. C. Sutton, D. Bhatnagar, M. I. Mackness, F. Creed, et al. 1995. "Coronary Risk Factors in People from the Indian Subcontinent Living in West London and Their Siblings in India." The Lancet. doi:10.1016/S01406736(95)90398-4.

Sadat, Sedigheh. 2012. "Predictors of Cervical Cancer Screening: An Application of Health Belief Model.” In Topics on Cervical Cancer With an Advocacy for Prevention. doi:10.5772/27886.

Scholes, Shaun. 2017. Health Survey for England 2016 Physical Activity in Adults. NHS Digital.

Sidhu, Manbinder S., Laura Griffith, Kate Jolly, Paramjit Gill, Tom Marshall, and Nicola K. Gale. 2016. "Long-Term Conditions, Self-Management and Systems of Support: An Exploration of Health Beliefs and Practices within the Sikh Community, Birmingham, UK.” Ethnicity and Health. doi:10.1080/13557858.2015.1126560.

Stuckey, Heather L, Cheryl Dellasega, Nora J Graber, David T Mauger, Irina Lendel, and 
Robert a Gabbay. 2010. "Diabetes UK and South Asian Health Foundation

Recommendations on Diabetes Research Priorities for British South Asians." Diabetes. doi:10.1016/j.cct.2009.03.002.Diabetes.

Taymoori, Parvaneh, and Tanya Berry. 2009. “The Validity and Reliability of Champion's Health Belief Model Scale for Breast Cancer Screening Behaviors among Iranian Women." Cancer Nursing. doi:10.1097/NCC.0b013e3181aaf124.

Tongco, Ma Dolores C. 2007. "Purposive Sampling as a Tool for Informant Selection." Ethnobotany Research and Applications. doi:10.17348/era.5.0.147-158.

Triandis, Harry C., Xiao Ping Chen, and Darius K.S. Chan. 1998. "Scenarios for the Measurement of Collectivism and Individualism." Journal of Cross-Cultural Psychology. doi:10.1177/0022022198292001.

Sidhu, Manbinder S., Laura Griffith, Kate Jolly, Paramjit Gill, Tom Marshall, and Nicola K. Gale. 2016. "Long-Term Conditions, Self-Management and Systems of Support: An Exploration of Health Beliefs and Practices within the Sikh Community, Birmingham, UK.” Ethnicity and Health. doi:10.1080/13557858.2015.1126560.

Singh, Jasjit. 2010. "Head First: Young British Sikhs, Hair, and the Turban.” Journal of Contemporary Religion. doi:10.1080/13537901003750894.

Sohi, Khushbeen Kaur, Purnima Singh, and Krutika Bopanna. 2018. "Ritual Participation, Sense of Community, and Social Well-Being: A Study of Seva in 
the Sikh Community." Journal of Religion and Health. doi:10.1007/s10943-0170424-y.

Stuckey, Heather L, Cheryl Dellasega, Nora J Graber, David T Mauger, Irina Lendel, and Robert a Gabbay. 2010. "Diabetes UK and South Asian Health Foundation Recommendations on Diabetes Research Priorities for British South Asians." Diabetes. doi:10.1016/j.cct.2009.03.002.Diabetes.

Thandi, Shinder S. 2018. "Educated Millennial Sikhs: Higher Education, Social Mobility and Identity Formation among British Sikh Youth.” Sikh Formations: Religion, Culture, Theory. doi:10.1080/17448727.2018.1485377.

Unnikrishnan, Ranjit, Prasanna Kumar Gupta, and Viswanathan Mohan. 2018. "Diabetes in South Asians: Phenotype, Clinical Presentation, and Natural History." Current Diabetes Reports. doi:10.1007/s11892-018-1002-8.

Varghese, Suja, and Robin Moore-Orr. 2002. "Dietary Acculturation and Health Related Issues of Indian Immigrant Families in Newfoundland." Canadian Journal of Dietetic Practice and Research. doi:10.3148/63.2.2002.72.

WHO. 2010. "World Heath Statistics: 2010.” World Heath Organization. doi:ISBN 9789241565257. 
Williams, Emily D., Emmanuel Stamatakis, Tarani Chandola, and Mark Hamer. 2011.

"Assessment of Physical Activity Levels in South Asians in the UK: Findings from the Health Survey for England." Journal of Epidemiology and Community Health. doi:10.1136/jech.2009.102509. 